\title{
Heat transfer in soft nanoscale interfaces: the influence of interface curvature
}

\author{
Anders Lervik ${ }^{1,2}$, Fernando Bresme $\stackrel{1,3, *}{ }$, Signe Kjelstrup ${ }^{2,3,4}$ \\ ${ }^{1}$ Department of Chemistry, Imperial College London, SW7 2AZ, UK \\ 2 Department of Chemistry, Norwegian University of Science and Technology, \\ Trondheim, Norway \\ ${ }^{3}$ on leave: Centre for Advanced Study at the Norwegian Academy of \\ Sciences and Letters, \\ Oslo, Norway \\ ${ }^{4}$ Department of Process and Energy, TU Delft, Delft, Netherlands
}

\author{
Received XXXXth Month, 200X \\ Accepted XXXXth Month, 200X \\ DOI: $10.1039 /$
}

We investigate using transient non-equilibrium molecular dynamics simulations, heat transfer through nanometer scale interfaces consisting of $n$-decane (2-12 $\mathrm{nm}$ diameter) droplets in water. Using computer simulation results of the temperature relaxation of the nanodroplet as a function of time we have computed the thermal conductivity and the interfacial conductance of the droplet and the droplet-water interface respectively. We find that the thermal conductivity of the $n$-decane droplets is insensitive to droplet size, whereas the interfacial conductance shows a strong dependence with the droplet radius. We rationalize this behavior in terms of a modification of the $n$-decane-water surface tension with droplet curvature. This enhancement in interfacial conductance would contribute, in the case of a suspension, to an increase in the thermal conductivity with decreasing particle radius. This notion is consistent with recent experimental studies of nanofluids. We also investigate the accuracy of different diffusion equations to model the temperature relaxation in non stationary non equilibrium processes. We show that the modeling of heat transfer across a nanodroplet-fluid interface requires the consideration of the thermal conductivity of the nanodroplet as well as the temperature discontinuity across the interface. The relevance of this result in diffusion models that neglect thermal conductivity effects in the modeling of the temperature relaxation is discussed.

\section{Submitted to the Emerging Investigators Issue}

\section{Introduction}

Nanoscale interfaces offer tremendous opportunities to design high performance materials. A paradigm of nanoscale interface is a nanoparticle suspension, where the solvent-nanoparticle interface has a typical size of the order of $10^{2}-10^{3} \mathrm{~nm}^{2}$. There is currently a vigorous research

\footnotetext{
*Corresponding author: f.bresme@imperial.ac.uk
} 
activity on nanoparticle interfacial related problems. Due to their small dimension the nanoparticles properties depart significantly from the ones observed in macroscopic materials. This feature can be exploited to make two and three dimensional materials with enhanced mechanical, optical and magnetic properties [1]. It is therefore of great interest to understand the stability and general behavior of nanopartices in different environments and dimensions, 2D - monolayers and 3D - suspensions. The behavior of 3D suspensions can be understood in many cases in terms of well accepted theories. On the other hand our knowledge of the interactions organizing 2D assemblies, particularly at fluid interfaces, is still incomplete [2].

Nanoparticles can strongly modify the properties of suspensions. This notion is being exploited to design high performance materials, nanofluids, with uses in thermal managament problems. This is motivating a number of studies aimed to understanding thermal transport in systems involving nanoscale interfaces. Recent reviews [3] have addressed this problem in systems where heat is transported by thermal vibrations or phonons. This situation is relevant to many nanomaterials consisting of metallic or semiconductor crystallites coated with organic layers in direct contact with a solution. The interface between the material, e.g., nanoparticle, and the solution, becomes increasingly important in the nanoscale. Hence, it is expected that for very small particles, few nm diameter, the thermal transport will be strongly dependent on the properties of the nanoscale interfaces, and therefore, it will be different from heat transfer in macroscopic systems, where interfaces become less relevant as compared with bulk effects [4, 5]. Understanding the mechanisms that control the heat transport in these situations, provides a route to modify the interface properties with a view to improve the properties of materials. This information can be important to design efficient materials for thermoelectric energy conversion, as well as medical applications concerned with drug delivery and cancer therapy treatments based on local heating of nanoparticles [6].

Kapitza [7] noted that the interface between a solid and a liquid has a thermal resistance, which can be understood considering the acoustic mismatch of the two materials. This is a general property that appears when two different phases are put in contact and therefore an interface is created. A practical implication of such resistance is the existence of a temperature "jump" across the interface. The magnitude of this temperature jump is an intrinsic property of the two phases in contact, and it is well known in the area of non equilibrium thermodynamics of heterogeneous systems [8] This theory defines surface resistivities in terms of surface variables. For instance, it is assumed that the transfer coefficients for evaporation of water depend on the surface temperature, but are independent of the temperature of adjacent bulk phases. This notion provides a route to define resistivities in terms of single interfacial properties, e.g, the surface tension.

The thermal transport of nanomaterials involving planar interfaces $[9,10]$ in solution, has been quantified experimentally and through computer simulations. These investigations report interfacial conductances (the inverse of the thermal resistance) of the order of $10-10^{2} \mathrm{MW} /\left(\mathrm{m}^{2}\right.$ $\mathrm{K})$. This represents an enormous variation in conductances. These differences among materials may be partially interpreted in terms of the vibrational coupling between the material in question and the solvent. Other studies have focused on small interfaces, with complex shapes as in biomolecules $[11,12,13,14]$, or spherical (nanoparticles) and cylindrical (nanotubes) shapes $[15,16,17,18,19,20,21,22]$. Similar interfacial conductances, $10^{2} \mathrm{MW} /\left(\mathrm{m}^{2} \mathrm{~K}\right.$ are reported for these cases, with the exception of the small values reported for nanotube suspensions [23] , $12 \mathrm{MW} /\left(\mathrm{m}^{2} \mathrm{~K}\right)$, and AuPd nanoparticles dispersed in toluene, $5 \mathrm{MW} /\left(\mathrm{m}^{2} \mathrm{~K}\right)$. The origin of this later observation is not well understood. Moreover, some experiments have reported a dependence of the thermal conductivity of nanoparticle suspensions with particle radius. The thermal conductivity apparently increases with decreasing particle radius [24, 25]. Several ideas have been put forward to rationalize this observation, including, the modification of the properties of the nanoparticle-solvent interface, and collective structural (clustering) and dynamical 
(particle diffusion) effects [24].

In our view this radius dependence, if proved to be present, may indicate a physical effect that goes beyond a pure dependence with chemical composition. Size effects have been reported in case of water, one of the main solvents used in nanofluid research. It is known that the properties of interfacial water are sensitive to the solute size and interface curvature, which results in a measurable dependence of the solvation free energy with solute diameter. Moreover, under non equilibrium conditions, e.g., in the presence of temperature gradients such as those considered in experiments and simulations, water may adopt a preferred orientation, an effect that may lead to strong electrostatic fields when considering nanoscale dimensions[26]. These observations show that new effects may appear in nanoscale systems. We believe that an understanding of the factors controlling the dependence of the interfacial conductance with interface curvature, could provide an explanation to the observed enhancement of the thermal conductivity in nanofluids. Investigating the dependence of the thermal conductance of an interface with interface curvature is far from trivial, since many effects, chemical composition, surface heterogeneity, etc, may influence the analysis. In this paper we have investigated this dependence by performing transient non equilibrium molecular dynamics simulations. We have considered $n$-decane nanodroplets with diameters 2-12 nm, immersed in water. This soft interface provides a good control of the droplet radius and sphericity, as well as uniform interface composition and interfacial smoothness. Overall, our results show a strong dependence of the interfacial conductance with interface curvature.

\section{The transient non equilibrium molecular dynamics method}

The computation of the interfacial conductance requires the explicit simulation of the interface. The conductance can be extracted from a non equilibrium molecular dynamics (NEMD) simulation, whereby a temperature gradient is imposed through the interface. Several methods have been proposed to perform such simulations $[27,28,29,30]$. These methods require long simulation times to attain the stationary state, and they have been used to compute the interfacial conductance [10] of water-organic interfaces, and the interfacial resistivity [8, 31] of liquid-vapor interfaces. As we will discuss later, the conductance and resistivity computed in different studies are closely connected to each other, although no such connection has been made in the literature before.

In addition to stationary NEMD method, non stationary NEMD or transient NEMD methods have been proposed. The transient NEMD method considers an explicit interface that separates the material of interest and the surrounding fluid. The material and the fluid are initially at different temperatures. The method requires the analysis of the temperature relaxation with time $[11,12]$. A fitting of the time dependent temperature to a diffusion equation, provides a route to estimate the interfacial conductance [23] and potentially the thermal conductivity [32]. Most studies have modeled the temperature relaxation as a first order process, but other other approaches are possible. In the following we discuss the three main approaches we have investigated in this work.

\subsection{Model A: Lumped model}

In this model the temperature of the material is assumed to be uniform during the relaxation process. This is equivalent to assume that the thermal conductivity of the material is infinite. This may provide a good approximation to model situations where the thermal conductivity of the material and the surrouding fluid are very different. An example could be a solid-water interface, where the solid can attain thermal conductivities of the order of $10^{3} \mathrm{~W} /(\mathrm{K} \mathrm{m})$ as compared with 
$10^{-1} \mathrm{~W} /(\mathrm{K} \mathrm{m})$ for water. This model also predicts temperature discontinuities at the materialfluid interface.

The temperature relaxation is modeled through the following equation [33],

$$
C_{p} \frac{d T}{d t}=-A G\left[T(t)-T_{f}\right]=-A J_{q}
$$

where $C_{p}$ and $A$ represent the isobaric heat capacity (in $J / K$ ) and surface area of the material, $G$ the thermal conductance of the surface and $J_{q}$ the heat flux normal to the surface.

The solution to this equation is given by:

$$
\frac{T(t)-T_{f}}{T_{i}-T_{f}}=\exp \left(-\frac{t}{\tau}\right)
$$

where the relaxation constant is defined by, $\tau=C_{p} /(A G)$, being $T_{i}$ is the initial temperature of the material and $T_{f}$ the final temperature, equal to the temperature of the surrounding fluid. Figure 1 shows the solution of this equation as a function of time for a representative example. A fitting of the temperature relaxation to equation (2) provides an estimate of the interfacial conductance, $G$. The Lumped models is probably the most popular approach in the analysis of simulation results, but this model does not include information of the thermal conductivity of the material. The thermal conductivity can be incorporated in the diffusion equation for heat conduction using an alternative diffusion equation, which we discuss in the following section.

\subsection{Model B}

This model includes the thermal conductivity of the material in the solution of the diffusion equation, and assumes that the temperature change across the interface is continuous. We shall use this model as an extreme case, that justifies the use of more sophisticated, models (Model C, see below). The equation describing this process is given by [33]:

$$
\rho c_{p} \frac{\partial T}{\partial t}=-(\nabla \cdot \mathbf{q})=\kappa \nabla^{2} T
$$

where $\rho$ is the density of the material and $c_{p}$ is the isobaric specific heat capacity per unit mass (in $J /(k g K))$. This model assumes 1 ) that Fourier's law is valid, and 2) that the material is homogenous regarding its thermal conductivity $\kappa$. For a spherical droplet like the one investigated in this work, the heat flux at a particular location depends on the distance to the center of the particle, therefore eqn. (3) can be simplified to [34],

$$
\frac{\rho c_{p}}{\kappa} \frac{\partial(r T(r, t))}{\partial t}=\frac{\partial^{2}(r T(r, t))}{\partial r^{2}}
$$

where $r<R$ is the radial distance to the center of mass of the droplet and $T(r, t)$ is the temperature at radius $r$ and time $t>0$. The solution to this equation can be obtained by impossing the initial condition, $T(r, t=0)=T_{i}$ and the boundary condition, $T(r=R, t)=T_{f}(t)$, where $T_{f}(t)$ is the temperature of the surrounding fluid, which is independent of the distance to the interface. The general solution to this equation can be found in refs. [33, 34]. Here, we are interested in the solution corresponding to a constant temperature of the surrounding fluid. This situation is valid when the heat capacity of the fluid is significantly higher than that of the material, so that the temperature of the solvent can be considered constant through the relaxation process. It turns out that the solution of the diffusion equation for this situation can be simplified if one removes the spatial dependence of the temperature inside the particle, by averaging such temperature over the whole droplet volume. Using this approach the solution to the equation becomes [34], 


$$
\frac{\langle T(r, t)\rangle_{V}-T_{f}}{T_{i}-T_{f}}=\frac{T(t)-T_{f}}{T_{i}-T_{f}}=\frac{6}{\pi^{2}} \sum_{n=1}^{\infty} \frac{1}{n^{2}} \exp \left(\frac{-n^{2} \pi^{2} t}{\tau}\right),
$$

where $\langle T(r, t)\rangle_{V}$ represent the average temperature of the droplet. Figure 1 shows an example of the general solution (see $[33,34]$ ). In this case the thermal conductivity of the droplet can be obtained by fitting the temperature relaxation to eqn. (5), which provides a route to estimate the relaxation time, $\tau$. The thermal conductivity is then given by:

$$
\kappa=\frac{\rho c_{p} R^{2}}{\tau}=\frac{3 C_{p}}{4 \pi R \tau} .
$$

The model discussed above, assumes that the temperature changes continuously across the interface between the material and the surrounding fluid. As mentioned in the introduction the acoustic mismatch of the materials will result in a temperature jump at the interface. Hence, model $\mathrm{B}$ is expected to be inaccurate when this mismatch is significant. This drawback can nonetheless be solved by introducing another model that incorporates both, the thermal conductivity of the material and the temperature jump.

\subsection{Model C}

In this model we include the thermal conductivity of the material and also we take into account the temperature discontinuity at the interface, therefore modelling the acoustic mismatch. The diffusion equation describing this situation is given by,

$$
-\left.\kappa \frac{\partial T}{\partial r}\right|_{r=R}=G\left(T(r=T, t)-T_{f}\right)
$$

where again the temperature of the surrounding fluid is assumed to be constant. Considering the initial condition, $T(r, t=0)=T_{i}$, the solution to this equation is given by [33],

$$
\frac{T(r, t)-T_{f}}{T_{i}-T_{f}}=4 \sum_{n=1}^{\infty} \frac{\sin \left(\lambda_{n}\right)-\lambda_{n} \cos \left(\lambda_{n}\right)}{2 \lambda_{n}-\sin \left(2 \lambda_{n}\right)} \exp \left(-\frac{\lambda_{n}^{2} t}{\tau}\right) \frac{R}{\lambda_{n} r} \sin \left(\frac{\lambda_{n} r}{R}\right)
$$

where

$$
1-\lambda_{n} \cot \left(\lambda_{n}\right)=\frac{G R}{\kappa}=\mathrm{Bi}
$$

where $\mathrm{Bi}$ is the Biot number.* A typical solution of model $\mathrm{C}$ is given in Fig. 1. In this paper the Biot number measures the ratio of the thermal resistance in the material and the thermal resistance of the interface [33]. Hence, for $\mathrm{Bi} \ll 1$ one recovers model $\mathrm{A}$, whereas for $\mathrm{Bi} \gg 1$ one recovers model $\mathrm{B}$. This means that the simple first order approximation (model A) would be valid only when the thermal resistance of the interface dominates.

Considering again the average temperature of the material instead of the spatial dependent expression, one can arrive to a more compact solution for the temperature relaxation with time,

$$
\frac{\langle T(r, t)\rangle_{V}-T_{f}}{T_{i}-T_{f}}=\frac{T(t)-T_{i}}{T_{i}-T_{f}}=6 \sum_{n=1}^{\infty} \frac{1}{\lambda_{n}^{3}} \frac{\left(\sin \left(\lambda_{n}\right)-\lambda_{n} \cos \left(\lambda_{n}\right)\right)^{2}}{\lambda_{n}-\sin \left(\lambda_{n}\right) \cos \left(\lambda_{n}\right)} \exp \left(\frac{-\lambda_{n}^{2} t}{\tau}\right),
$$

The solution can further be simplified by retaining only the first term in eqn. (10)[33],

${ }^{*}$ The Biot number is usually defined using the convective heat transfer number instead of the conductance, but the equations are mathematically equivalent. Therefore the term "Biot number" is used in the remainder of the paper. 


$$
\frac{T(t)-T_{i}}{T_{i}-T_{f}}=6 \frac{1}{\lambda_{1}^{3}} \frac{\left(\sin \left(\lambda_{1}\right)-\lambda_{1} \cos \left(\lambda_{1}\right)\right)^{2}}{\lambda_{1}-\sin \left(\lambda_{1}\right) \cos \left(\lambda_{1}\right)} \exp \left(\frac{-\lambda_{1}^{2} t}{\tau}\right),
$$

which represents a good approximation for $t>0.2 \tau$. This equation is more convenient for the analysis of the simulation results and it is the one we have employed in this work. A fitting of the temperature relaxation to this equation provides a route to compute both the thermal conductance of the interface and the thermal conductivity of, in our case, the alkane nanodroplet. As a general comment we can conclude that models $\mathrm{A}$ and $\mathrm{C}$ should be applicable to interfaces, since they include the possibility of a discontinuity of the temperature across the boundary (see Fig. 1). The performance of these three models is discussed in the results section.

\subsection{Non equilibrium simulations}

The alkane molecules, $n$-decane, were modeled using the TraPPE forcefield [35], which considers a united atom representation of the $\mathrm{CH}_{3}$ and the $\mathrm{CH}_{2}$ groups. Water was modeled using the SPC/E model [36]. The Coulombic interactios were truncated at $1.5 \mathrm{~nm}$ using a switching function at 1.3 $\mathrm{nm}$. The van der Waals interactions were cut-off at $1.7 \mathrm{~nm}$. We have recently considered this set up to investigate the properties of the oil-water interface. We showed that this interface undergoes very weak short-ranged fluctuations at ambient temperature [37, 38]. All the simulations were performed using the GROMACS simulation package [39].

The general procedure used in the transient non equilibrium simulations was as follows. The $n$ decane nanodroplets were immersed in the center of a water spherical shell, which was surrounded by vacuum. The relaxation simulations were performed without periodic boundary conditions, and both the linear and angular momentum were removed to avoid violation of energy equipartition [40]. Due to the low vapor pressure of water we did not observe any significant water evaporation during the simulations. The water shell around the decane of the nanodroplet was always thick enough, about $2.5 \mathrm{~m}$, to ensure there was bulk water away from the interfacial region (see Figure [?]). We checked that the properties investigated in this work, thermal conductivity and interfacial conductance were insensitive within statistical error to the thickness of the water shell. We considered alkane nanodroplet of diameters, 2 to $12 \mathrm{~nm}$, in order to assess the effect of the droplet size on the interfacial conductance. Before analyzing the relaxation process we equilibrated the whole system at constant temperature, $300 \mathrm{~K}$. After the initial equilibration two Berendsen thermostats [41] were applied, one to the alkane droplet and the other to the water shell, typically for $10 \mathrm{ps}$, in order to allow the temperatures of the alkanes and water to fluctuate around their corresponding target values, $T_{i}=400 \mathrm{~K}$ and $T_{f}=300 \mathrm{~K}$. After this equilibration period, the thermostat of the alkane nanodroplet was removed and the system was allowed to relax. The temperature of water was maintained constant during the relaxation process using a Berendsen thermostat. We found that the results were insensitive within statistical error to the values employed for the temperature coupling constant, which was set to $0.1 \mathrm{ps.} \mathrm{The} \mathrm{temperature}$ relaxation process was followed for 200 ps in most cases, using 2 fs as timestep. The temperature for the alkane droplet was computed every time step during the relaxation process through the equipartition principle expression. The variation of the temperature with time was recorded and analyzed using the diffusion models discussed above (section 2). All the results reported in this work were obtained from an analysis of three independent runs.

The analysis of the temperature relaxation using the models $\mathrm{A}, \mathrm{B}$ and $\mathrm{C}$, was performed through a least squares fit for model $\mathrm{A}$ and the Levenberg-Marquadt algorithm for models $\mathrm{B}$ and $\mathrm{C}[42]$. For model B the first 10 terms in the sum were considered. For model $\mathrm{C}$ we used equation (11), where only the first term in the series is retained. We made initial tests using equation (10), retaining the first five terms in the series, but we found this approach is too sensitive to the 
fitting parameters and the fluctuations in the temperature relaxation, rendering estimates for the thermal conductance that are not physical. We found that equation (11) provides a robust approach to fit the simulation data if all the data $t>0$ are included in the fitting. We used this approach in subsequent analyses.

The computation of the thermal conductance using the models discussed above requires to know the heat capacity of the system. The heat capacity of each nanodroplet was estimated from constant temperature runs of the alkane-water system. We note that because there are not periodic boundary conditions in our simulations, the nanodroplet is allowed to change its volume, resembling a constant pressure situation. The heat capacity was estimated from direct numerical differentiation of the internal energy using systems with temperatures around the temperature of interest. The computation of the diffusivity and the conductivity also requires the radius of the alkane nanodroplet. This was estimated from the radial density profile of the alkane molecules, which was fitted to the expression:

$$
\rho(r)=\frac{1}{2}\left(\rho_{a}+\rho_{b}\right)+\frac{1}{2}\left(\rho_{a}-\rho_{b}\right) f(r-R),
$$

where $\rho_{a}$ and $\rho_{b}$ are the densities $n$-decane in the droplet and in the water phase. We found that the density in the latter case was always zero. The function, $f(r-R)$, can be represented through,

$$
f(r-R)=\tanh (w(r-R)),
$$

or

$$
f(r-R)=\operatorname{erf}(w(r-R)),
$$

where $w$ is a measurement of the thickness of the interface and $R$ is the radius of the droplet. Fitting of the profiles to either eqn. (13) or eqn. (14) provides the same estimates for $R$ within statistical uncertainty. Figure 2, shows a representative density profile for a decane nanodroplet in water at $300 \mathrm{~K}$. We note that the density of the droplet, as expected for a curved surface, is slightly higher than the experimental coexistence density of $n$-decane for a planar interface.

\section{Results and Discussion}

Before we start the discussion of the temperature relaxation results, it is convenient to use the Biot (Bi) number to assess the applicability of the models described above to investigate nanoscale interfaces. For a nanometer size sphere of radius, $R \approx 1 \mathrm{~nm}$, a thermal conductance of the order of $G \approx 10^{2} \mathrm{MW} /\left(\mathrm{m}^{2} \mathrm{~K}\right)$, and a thermal conductivity of the order of $\kappa \approx 0.1 \mathrm{~W} /(\mathrm{m} \mathrm{K})$, which is typical of many hydrocarbon liquid compounds, would give $\mathrm{Bi} \approx 1$. This simple calculation indicates that in the case of nanomaterials and liquids with small thermal conductances, the heat transfer resistance in the material and the interface are of the same order. Hence, both have to be included in order to interpret the temperature relaxation process. From this perspective we expect that model $\mathrm{C}$ should be the most accurate describing the temperature relaxation of the nanoscale interfaces investigated in this work.

Figure 3 shows a representative temperature relaxation of one $n$-decane nanodroplet in water, along with the corresponding fit to the the three different models discussed in the preceding section. Numerical results are compiled in table 1 . We find that model $\mathrm{C}$ as implemented in equation (11) provides a better representation of the temperature relaxation over the whole time interval. This is particularly clear at short relaxation times, (0-20 ps, see inset in Fig. 3), where models A and B deviate from the simulation results. Comparing models A and B, the latter seems to be the less accurate to fit our simulation results. The nature of the approximations enclosed 
in the different models is reflected in the transport coefficients estimated from the fittings. As a matter of fact model $\mathrm{C}$ tends to predict thermal conductances that are larger by a factor of two, than those obtained with model A. The discrepancies between models $\mathrm{C}$ and $\mathrm{B}$ are more dramatic. We find that that the thermal conductivity and diffusivity of the $n$-decane nanodroplets, predicted by model $\mathrm{C}$ ranges from $0.07-0.16 \mathrm{~W} /(\mathrm{K} \mathrm{m})$ and $5-15 \AA^{2} /$ ps respectively (see table 1 ), which are of the order of the experimental results for $n$-decane $8.0 \AA^{2} /$ ps at $300 \mathrm{~K}$ [43]. On the other hand model $\mathrm{B}$ predicts thermal conductivities, 0.03-0.05 W/(K m) and diffusivities, $2-5 \AA^{2} / \mathrm{ps}$ (see table 1) that are too low by one order of magnitude, showing that this is clearly an inadequate model to investigate heat transfer in nanoscale interfaces.

Figures 4 shows the dependence of the thermal conductance, $G$, with the $n$-decane droplet diameter. The interface resistance to heat flow as measured by $G$ exhibits a strong dependence with droplet radius. The interfacial conductance is reduced by a factor of two upon an increase in interface area of $4 \pi R^{2} \approx 1.3 \times \mathrm{nm}^{2}$ to $\approx 4.5 \times 10^{2} \mathrm{~nm}^{2}$, hence the nanodroplet-water interface becomes a worse conductor as the droplet radius, $R$, increases. Both, models $\mathrm{A}$ and $\mathrm{C}$ are consistent in predicting this general trend. We note that a decrease in interfacial conductance would contribute to a decrease in the overall thermal conductivity of a nanofluid (nanoparticle suspension). This observation is compatible with recent observations of particle suspensions, which have reported an increase in the thermal conductivity with a decrease in particle diameter [24].

Unlike model A, model $\mathrm{C}$ incorporates the thermal conductivity of the nanodroplet as a variable. This provides the opportunity to assess a possible dependence of our results with this quantity. As shown in Figure 5 and table 1, the thermal conductivity shows small changes with droplet size, except for the smallest droplet investigated in our work. The thermal conductivities for the droplets $\left(1 / R<0.8 \mathrm{~nm}^{-1}\right.$ c.f. Fig. 5$)$ can be compared with literature data for $n$-decane. We find that the thermal conductivity of the TraPPE model investigated here agrees with simulation data of similar united atom models, which lie in the interval $0.1-0.137 \mathrm{~W} /(\mathrm{m} \mathrm{K})$ [45], and also show an excellent agreement with experimental data of $n$-decane, $0.132 \mathrm{~W} /(\mathrm{K} \mathrm{m})$ at $300 \mathrm{~K}[44,43]$. The interfacial conductances reported above, $100-250 \mathrm{MW} /\left(\mathrm{m}^{2} \mathrm{~K}\right)$ are significantly larger than the ones reported by other authors for "planar" n-octane-water interface, $65 \mathrm{MW} /\left(\mathrm{m}^{2} \mathrm{~K}\right)[10]$. We note that these data were obtained using a non equilibrium stationary approach, different to the transient non equilibrium method employed by us. A planar interface represents the limit of a droplet of infinite radius in our investigation. An extrapolation of our data for model $\mathrm{C}$ to that limit shows an excellent agreement with the data published for the n-octane-water system, indicating that the origin of the differences between both results is the curvature of the interface. This result also shows that model A, which is widely used in computer simulation studies and experiments, tends to underestimate the interfacial conductance. A linear extrapolation of the data for model A gives a conductance of the order of $20 \mathrm{MW} /\left(\mathrm{m}^{2} \mathrm{~K}\right)$, similar to values reported for nanotube-octane interfaces (nanotube radius $0.35 \mathrm{~nm}$ ), which were obtained using a first order relaxation (model A) approach.

From our results for the interfacial conductance, nanodroplet radius and thermal conductivities, we have estimated the Biot number (c.f. eqn. (9)) for the different systems investigated in this work. We find that all the values are in the range, $1<\mathrm{Bi}<6$, showing that the nanoscale systems investigated here do not fulfill the conditions $\mathrm{Bi} \ll 1$ or $\mathrm{Bi} \gg 1$, which are necessary for models $\mathrm{A}$ or B to apply.

Our results point to a purely interfacial effect as the one responsible for the reduction of the interfacial conductance with droplet diameter, or in other words, interface curvature. In the following we try to rationalize this idea in terms of the surface tension of the interface. It is known that the surface tension of a spherical fluid interface decreases with droplet radius. To first order, the change in surface tension can be described in terms of Tolman's equation [46], 


$$
\gamma(R)=\gamma_{\infty}\left(1-\frac{2 \delta}{R}\right),
$$

where $\gamma_{\infty}$ is the surface tension of the planar interface and $\delta$ is the Tolman length, which for simple liquids is positive and increases as the temperature approaches the critical point [47]. The reduction of the surface tension of spherical interfaces with interface area, has been investigated in liquid-vapor [48], and also nanoparticle-liquid interfaces [49]. The latter study showed that the surface tensions follow approximately the linear dependence predicted by Tolman's equation. The reduction of the surface tension of liquid-vapour interfaces of simple liquids, such as Argon, can be significant, $\approx 50 \%$ for a liquid droplet of radius $\approx 1.2 \mathrm{~nm}[48]$. Similarly, in our case the surface tension of the $n$-decane nanodroplet should decrease with radius, making the interface softer for smaller particles.

This reduction in surface tension may explain the increase in interfacial conductance, because the conductance decreases with an increase in surface tension [8]. To prove this, it is convenient to introduce first the interfacial resistivity, $r_{q q}$. The derivation of this quantity relies on the notion that the interface can be see as a separate thermodynamics system. The interfacial resistivity can be defined, in absence of net mass flux, in terms of the heat flux, $J_{q}$,

$$
J_{q}=-\frac{1}{T_{1} T_{2} r_{q q}} \Delta T
$$

where $\Delta T$ is the temperature jump across the interface between the two materials " 1 " and " 2 ". Equation (16) is the Fourier's law for the interface, and it is very similar to the equation defining the interfacial conductance used here and in most publications dealing with heat transfer across interfaces,

$$
J_{q}=-G \Delta T,
$$

Hence, the conductance is given by $G=1 /\left(T_{1} T_{2} r_{q q}\right)$. Using stationary non equilibrium molecular dynamics simulations, it has been shown that the interfacial resistivity, $r_{q q}$, increases with the surface tension $[8,31]$, which results in a decrease of the thermal conductance, $G$. This decrease would be consistent with our results. These studies have focused on planar liquid-vapor interfaces, for which the estimated conductance (considering typical values of resisitivities for Lennard-Jones and octane interfaces) is of the order of $1 \mathrm{MW} /\left(\mathrm{K} \mathrm{m}^{2}\right)$, much smaller than the conductances observed in planar alkane-water interfaces, $65 \mathrm{MW} /\left(\mathrm{m}^{2} \mathrm{~K}\right)[10]$. This large difference in conductance is somewhat expected considering the difference in the acoustic mismatch of liquid-vapor and liquid-liquid interfaces.

\section{Conclusions}

We have investigated the dependence of the interfacial conductance of an alkane nanodropletwater interface as a function of nanodroplet diameter $(2-12 \mathrm{~nm})$, using transient non-equilibrum molecular dynamics simulations. We find a strong dependence in the interfacial conductance with nanodroplet diameter, i.e., interface curvature, showing that the interface becomes less resistant to heat transfer as the droplet becomes smaller. We explain the reduction in conductivity with increasing droplet size as a surface tension effect. The surface tension of the nanodroplets increases with the diameter. Increasing the surface tension results in a stiffer interface which has a lower conductance. Our result is consistent with stationary non equilibrum simulations of planar liquidvapor interfaces, which showed a clear dependence of the interfacial conductance with surface tension [8]. 
This observation can be important to understand thermal conductivity experiments in nanofluids. A reduction of the interfacial conductance would have an impact on the overall thermal conductivity of a nanoparticle suspension. According to our results the thermal conductivity should increase with a decrease in droplet size or increase of the interface curvature. This notion seems consistent with experimental observations of oxide nanoparticles in water (radius 6-20 nm), which have reported a significant increase in the thermal conductivity with decreasing particle diameter. It has been suggested that the increase in the thermal conductivity could be connected to a modification of the layering structure of the liquid around nanoparticles [24, 21]. We note that in our soft interfaces the density profile of water near the interface changes marginally with droplet diameter (for radii larger than $\approx 1 \mathrm{~nm}$ ). Despite this we still observe a significant reduction in interfacial conductivity. This shows that a modification of the interfacial conductance does not require a major change in the fluid interfacial structure.

We have assessed the accuracy of different diffusion equations to model the temperature relaxation of our transient non equilibrium simulations. We conclude that a model that incorporates the thermal conductivity of the material and the temperature discontinuity at the interface is the most adequate to model the heat transfer in the nanoscale soft interfaces investigated here. We have shown that for nanoscale interfaces the ratio of the heat transfer resistance in the material and the interface are of the same order, making necessary including the temperature discontinuity and the thermal conductivity in the model. Approaches widely used in the literature, which model the temperature relaxation as a first order process do not include information on the thermal conductivity. We find that these models provide a less accurate representation of the temperature relaxation and result in an underestimation of the interfacial conductances.

\title{
Acknowledgments
}

We would like to acknowledge the Imperial College High Performance Computing Service for providing computational resources.

\author{
Anders Lervik ${ }^{a}$, Fernando Bresme ${ }^{b}$ and Signe Kjelstrup ${ }^{c}$ \\ a Department of Chemistry, Imperial College London, London, SW7 2AZ, UK, and Department \\ of Chemistry, Norwegian University of Science and Technology, Trondheim, Norway E-mail: an- \\ ders.lervik@chem.ntnu.no \\ ${ }^{b}$ Department of Chemistry, Imperial College London, SW7, 2AZ, UK and Centre for Advanced \\ Study at the Norwegian Academy of Sciences and Letters, Oslo, Norway, E-mail:f.bresme@imperial.ac.uk. \\ ${ }^{c}$ Department of Chemistry, Norwegian University of Science and Technology, Trondheim, Nor- \\ way and Department of Process and Energy, TU Delft, Delft, Netherlands. On leave to: Cen- \\ tre for Advanced Study at the Norwegian Academy of Sciences and Letters, Oslo, Norway, E- \\ mail:.signe.kjelstrup@chem.ntnu.no
}




\section{References}

[1] G.A. Ozin, and A. Arsenault, Nanochemistry: A Chemical Approach to Nanomaterials, 2005, Cambridge: RSC Publishing.

[2] F. Bresme, and M. Oettel, J. Phys. Condens. Matter, 2007, 19, 413101.

[3] D.G. Cahill, W.K. Ford, K.E. Goodson, G.D. Mahan, A. Majumdar, H.J. Maris, R. Merlin and S.R. Phillpot, J. Appl. Phys., 2003, 93, 793.

[4] G. Chen, Nanoscale Energy Transport and Conversion: A Parallel Treatment of Electrons, Molecules, Phonons, and Photons, 2005, Oxford University Press, Oxford, UK.

[5] S. Volz, editor, Microscale and Nanoscale Heat Transfer, 2007, Springer, Berlin, Germany.

[6] P. K. Jain, I. H. El-Sayed, and M. A. El-Sayed, Nano Today, 2007, 2, 18.

[7] P.L. Kapitza, J. Phys. (Moscow), 1941, 4, 181.

[8] S. Kjelstrup and D. Bedeaux, Non-equilibrium Thermodynamics of Heterogeneous Systems, 2008, Series on Statistical Mechanics (World Scientific, Singapore), Vol. 16.

[9] Z. Ge, D.G. Cahill and P.V. Braun, Pys. Rev. Lett., 2006, 96, 186101.

[10] H. A. Patel, S. Garde, and P. Keblinski, Nano. Lett., 2005, 5, 2225.

[11] M. Tesch and K. Schulten, Chem. Phys. Lett., 1990, 169, 97.

[12] K. Blumhagen, I. Muegge and E.W. Knapp, Intl. J. Quantum. Chem., 1996 59, 271.

[13] X. Yu and D.M. Leitner, J. Chem. Phys., 2005, 122, 054902.

[14] D.M. Leitner, Annu. Rev. Phys. Chem., 2008, 59, 233.

[15] Y.C. Chiew and E.D. Glandt, Chem. Eng. Sci., 1987, 42, 2677.

[16] J.A. Eastman, S.U.S. Choi, S. Li, W. Yu, and L.J. Thompson, Appl. Phys. Lett., 2001, 78, 718.

[17] S.U.S. Choi, Z.G. Zhang, W. Yu, F.E. Lockwood, and E.A. Grulke, Appl. Phys. Lett., 2001, 79, 2252.

[18] Z. Ge, D. G. Cahill, and P. V. Braun, J. Phys. Chem. B, 2004, 108, 18870.

[19] J. C. Deak, Y. Pang, T.D. Sechler, Z. Wang, D.D. Dlott, Science, 2004, 306, 473.

[20] S. T. Huxtable, D. G. Cahill, S. Shenogin, P. Keblinski, Chem. Phys. Lett., 2005, 407, 129.

[21] H. Xie, M. Fujii and X. Zhang, Int. J. of Heat and Mass Trans., 2005, 48, 2926.

[22] S.A. Putnam, D.G. Cahill, P.V.Braun, Z.Ge and R.S. Shimmin, J. Appl. Phys., 2006, 99, 084308.

[23] S.T. Huxtable et. al., Nature Materials, 2003, 21, 731.

[24] P. Keblinski, S.R. Phillpott, S.U.S. Choi, and J.A. Eastman, Int. J. of Heat and Mass Trans., 2002, 45, 855. 
[25] H. E. Patel, S. K. Das, T. Sundararajan, A. S. Nair, B. George, and T. Pradeepa, Appl. Phys. Lett., 2003 83, 2931.

[26] F. Bresme, A. Lervik, D. Bedeaux and S. Kjesltrup, Phys. Rev. Lett., 2008, 101, 020602.

[27] T. Ikeshoji and B. Hafskjold, Mol. Phys., 1994, 81, 251.

[28] F. Bresme, B. Hafskjold, and I. Wold, J. Phys. Chem., 1996, 100, 1879.

[29] F.J. Muller-Plathe, J. Chem. Phys., 1997, 106, 6082.

[30] F. Bresme, J. Chem. Phys., 2001, 115, 7564.

[31] J.M. Simon, S. Kjelstrup, D. Bedeaux and B. Hafskjold, J. Phys. Chem. B, 2004, 108, 7186.

[32] R.J. Hulse, R.L. Rowley and W.V. Wilding, Int. J. of Thermophys., 2005, 26, 1.

[33] F. P. Incropera, D. P. DeWitt, T. L. Bergman, and A. S. Lavine, Fundamentals of Heat and Mass Transfer 2006, John Wiley \& Sons, Hoboken, USA, 6th edition.

[34] H. S. Carslaw and J. C. Jaeger, Conduction of Heat in Solids, 1959, Oxford University Press, Oxford, UK, 2nd edition

[35] M. G. Martin and J. I. Siepmann, J. Phys. Chem. B., 1998, 102, 2569.

[36] H.J.C. Berendsen, J.R. Grigera and T.P. Straatsma, J. Phys. Chem., 1987, 91, 6269.

[37] F. Bresme, E. Chacón, P. Tarazona and K. Tay, Phys. Rev. Lett., 2008, 101, 056102.

[38] F. Bresme, E. Chacón and P. Tarazona, Phys. Chem. Chem. Phys., 2008, 10, 4704.

[39] D. van der Spoel, E. Lindahl, B. Hess, G. Groenhof, A. E. Mark, and H. J. Berendsen, J. Comput. Chem., 2005 26, 1701.

[40] S. C. Harvey, R. K.-Z. Tan, and T. E. Cheatham III, J. Comput. Chem., 1998, 19, 726.

[41] H. J. C. Berendsen, J. P. M. Postma, W. F. van Gunsteren, A. DiNola, and J. R. Haak., J. Chem. Phys., 1984, 81, 3684.

[42] W. H. Press, S. A. Teukolsky, W. T. Vetterling, and B. P. Flannery, 2007, Numerical Recipes: The Art of Scientic Computing, Cambridge University Press, Cambridge, UK, 3rd edition.

[43] H. Watanabe and D. J. Seong, Int. J. Thermophys., 2002, 23, 337.

[44] M. J. Assael, E. Charitidou, C. A. Nieto de Castro, and W. A. Wakeham, Int. J. of Thermophys., 1987, 8, 663.

[45] D.K. Dysthe, A.H. Fuchs, and B. Rousseau, J. Chem. Phys., 2000, 112, 7518.

[46] R.C. Tolman, J. Chem. Phys., 1949, 17, 333.

[47] A. E. van Giessen and E. M. Blokhuis, J. Chem. Phys., 2002, 116, 302.

[48] S.M. Thompson, K.E. Gubbins, J.P.R.B. Walton, R.A.R. Chantry and J.S. Rowlinson, J. Chem. Phys., 1984, 81, 530.

[49] F. Bresme and N. Quirke, J. Chem. Phys., 1999, 110, 3536. 
Table 1: Thermal diffusivity (D), conductivity $(\kappa)$ and conductance $(\mathrm{G})$ of the decane nanodroplets as a function of droplet diameter. $T_{i}=400 \mathrm{~K}$, and $T_{f}=300 \mathrm{~K}$.

\begin{tabular}{lllllll}
\hline Radius $/ \mathrm{nm}$ & $N_{\text {dec }}$ & $N_{\text {wat }}$ & Model & $\mathrm{D} /\left(\AA^{2} / \mathrm{ps}\right)$ & $\kappa /(\mathrm{W} /(\mathrm{K} \mathrm{m}))$ & $\mathrm{G} /\left(\mathrm{MW} /\left(\mathrm{K} \mathrm{m}^{2}\right)\right)$ \\
\hline $1.11 \pm 0.01$ & 25 & 8666 & $\mathrm{~A}$ & - & - & $100 \pm 30$ \\
& & & $\mathrm{~B}$ & $2.0 \pm 0.4$ & $0.028 \pm 0.006$ & - \\
& & & $\mathrm{C}$ & $5 \pm 2$ & $0.07 \pm 0.02$ & $250 \pm 30$ \\
\hline $1.54 \pm 0.01$ & 60 & 8342 & $\mathrm{~A}$ & - & - & $110 \pm 30$ \\
& & & $\mathrm{~B}$ & $2.7 \pm 0.4$ & $0.033 \pm 0.004$ & - \\
& & & $\mathrm{C}$ & $12 \pm 5$ & $0.16 \pm 0.05$ & $170 \pm 40$ \\
\hline $2.54 \pm 0.02$ & 248 & \multirow{2}{*}{19397} & $\mathrm{~A}$ & - & - & $75 \pm 2$ \\
& & & $\mathrm{~B}$ & $3.8 \pm 0.2$ & $0.044 \pm 0.002$ & - \\
& & & $\mathrm{C}$ & $12 \pm 2$ & $0.14 \pm 0.02$ & $150 \pm 20$ \\
\hline $3.24 \pm 0.03$ & 500 & \multirow{2}{*}{17171} & $\mathrm{~A}$ & - & - & $70 \pm 4$ \\
& & & $\mathrm{~B}$ & $3.8 \pm 0.4$ & $0.041 \pm 0.004$ & - \\
& & & $\mathrm{C}$ & $15 \pm 6$ & $0.16 \pm 0.07$ & $90 \pm 20$ \\
\hline $4.10 \pm 0.04$ & \multirow{2}{*}{398} & 30538 & $\mathrm{~A}$ & - & - & $54 \pm 4$ \\
& & & $\mathrm{~B}$ & $4.4 \pm 0.4$ & $0.048 \pm 0.004$ & - \\
& & & $\mathrm{C}$ & $11.8 \pm 0.9$ & $0.13 \pm 0.01$ & $100 \pm 20$ \\
\hline $6.23 \pm 0.07$ & 3352 & 81001 & $\mathrm{~A}$ & - & - & $41 \pm 2$ \\
& & & $\mathrm{~B}$ & $5.1 \pm 0.1$ & $0.052 \pm 0.001$ & - \\
& & & $\mathrm{C}$ & $12 \pm 2$ & $0.12 \pm 0.02$ & $90 \pm 9$ \\
\hline
\end{tabular}


Fig. 1 Temperature relaxation of a spherical particle of radius $\mathrm{R}=3 \mathrm{~nm}$, initially at temperature $T_{i}$, as a function of distance to the center of the particle and for three different times. (a) model A (Lumped model) (eqn.(2)) (b) model B, where the thermal conductivity of the material is considered and the temperature is assumed to be continous across the boundary and (c) model $\mathrm{C}$, where the thermal conductivity is considered and the temperature is assumed to be discontinous across the boundary. The data correspond to the following conditions: $\kappa=0.15 \mathrm{~W} /(\mathrm{K} \mathrm{m}), G=100 \mathrm{MW} /\left(\mathrm{m}^{2} \mathrm{~K}\right)$, $C_{p}=200 \mathrm{~J} / \mathrm{mol} \mathrm{K}$ and $\rho=730 \mathrm{~kg} / \mathrm{m}^{3}$.

Fig. 2 Radial density profiles of the $n$-decane nanodroplets in water at $300 \mathrm{~K}$. The full line represents the fit of the decane density profile to equation (14). The decane nanodroplet contains 500 molecules and the water shell 17171.

Fig. 3 Temperature relaxation of an $n$-decane nanodroplet in water. The initial temperature is $400 \mathrm{~K}$ and the temperature of the surrounding fluid is $300 \mathrm{~K}$. The radius of the $n$-decane droplet containing 998 molecules is $4.1 \mathrm{~nm}$. The inset shows in detail the temperature relaxation in the interval 0-20 ps. The fitting of the simulation data to the three different models $\mathrm{A}, \mathrm{B}$ and $\mathrm{C}$ and the corresponding regression coefficient are also shown.

Fig. 4 Dependence of the thermal conductance with the nanodroplet size for models A (circles) and $\mathrm{C}$ (squares). The diamond represents an estimate of the thermal conductance of the octane-water planar interface using an stationary non equilibrium molecular dynamics approach[10].

Fig. 5 Dependence of the thermal conductivity with the nanodroplet size for model C (symbols). The dotted line represents the experimental thermal conductivity of $n$-decane at $300 \mathrm{~K}$ [44]. 


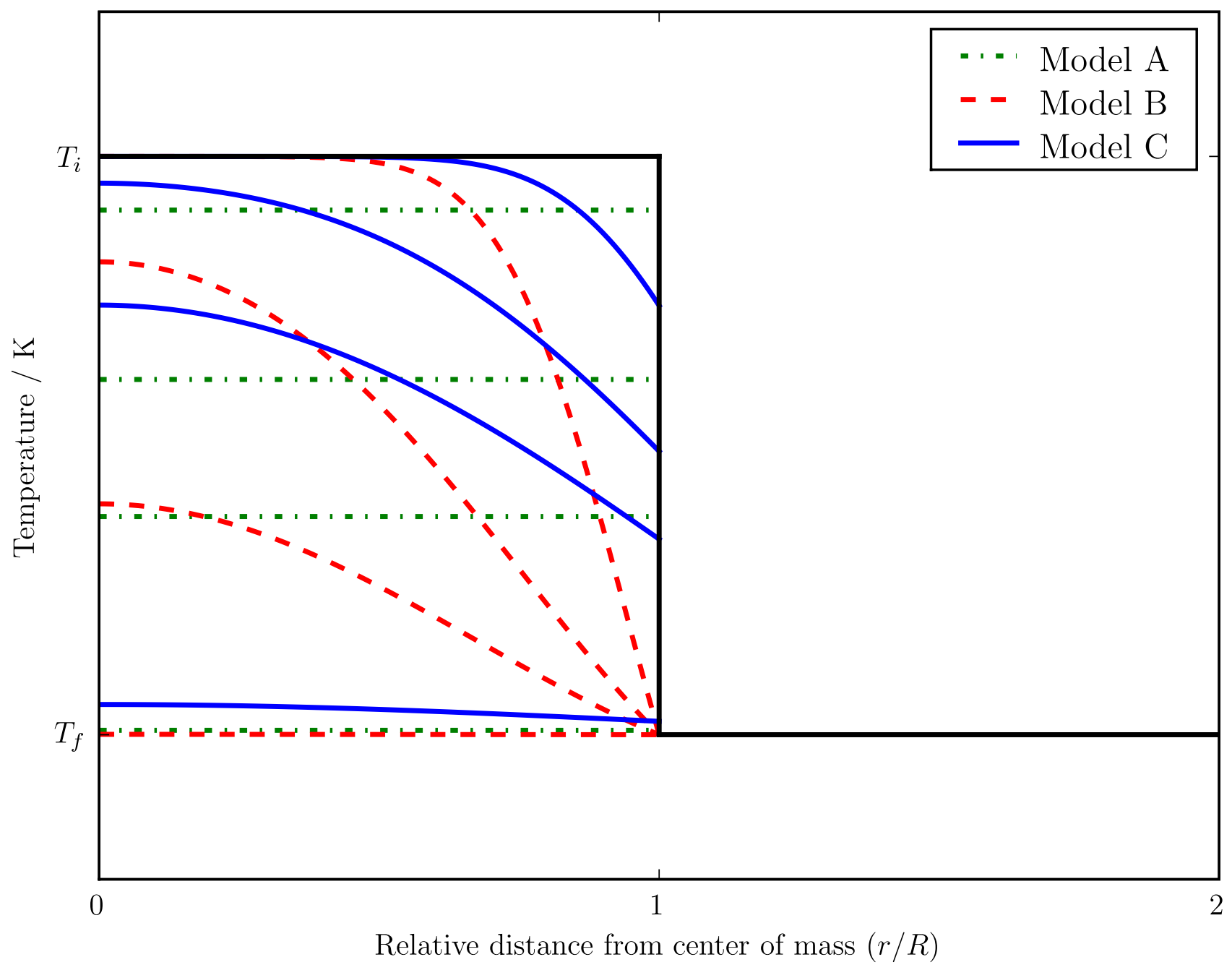

Figure 1: Temperature relaxation of a spherical particle of radius $\mathrm{R}=3 \mathrm{~nm}$, initially at temperature $T_{i}$, as a function of distance to the center of the particle and for three different times. (a) model A (Lumped model) (eqn.(2)) (b) model B, where the thermal conductivity of the material is considered and the temperature is assumed to be continous across the boundary and (c) model C, where the thermal conductivity is considered and the temperature is assumed to be discontinous across the boundary. The data correspond to the following conditions: $\kappa=0.15 \mathrm{~W} /(\mathrm{K} \mathrm{m})$, $G=100 \mathrm{MW} /\left(\mathrm{m}^{2} \mathrm{~K}\right), C_{p}=200 \mathrm{~J} / \mathrm{mol} \mathrm{K}$ and $\rho=730 \mathrm{~kg} / \mathrm{m}^{3}$. 


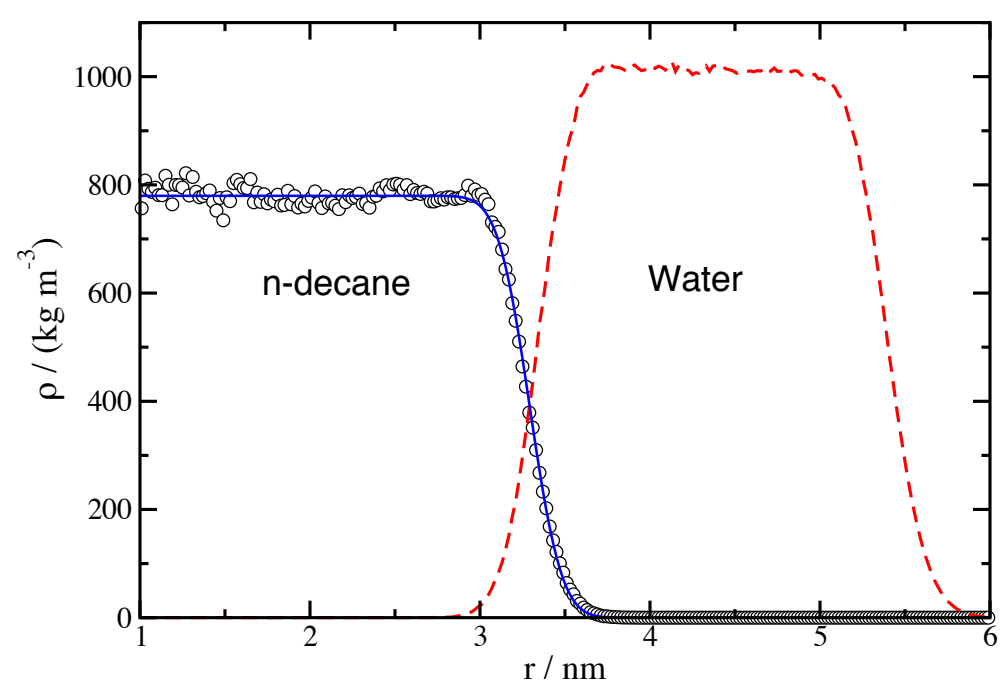

Figure 2: Radial density profiles of the $n$-decane nanodroplets in water at $300 \mathrm{~K}$. The full line represents the fit of the decane density profile to equation (14). The decane nanodroplet contains 500 molecules and the water shell 17171.

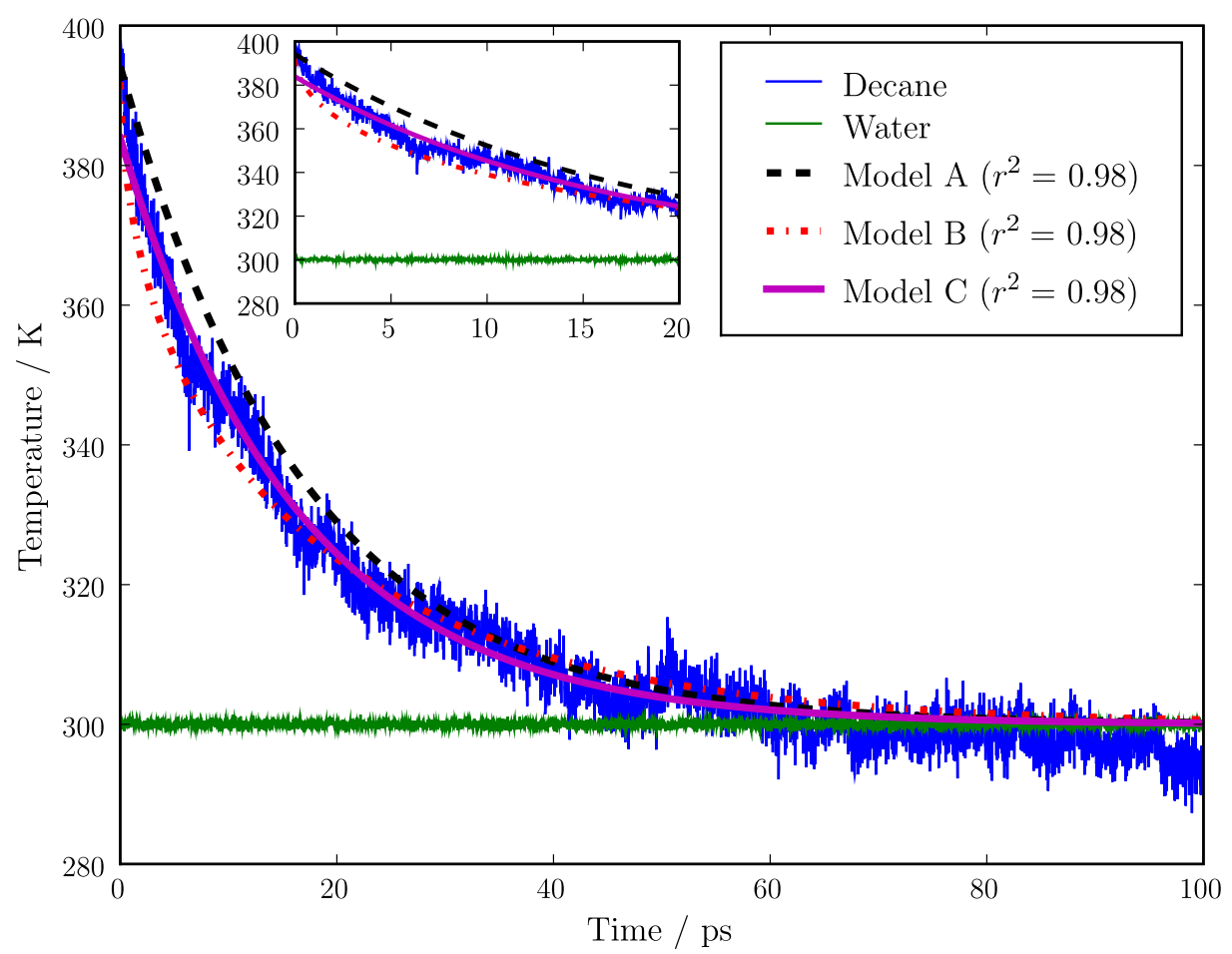

Figure 3: Temperature relaxation of an $n$-decane nanodroplet in water. The initial temperature is $400 \mathrm{~K}$ and the temperature of the surrounding fluid is $300 \mathrm{~K}$. The radius of the $n$-decane droplet containing 998 molecules is $4.1 \mathrm{~nm}$. The inset shows in detail the temperature relaxation in the interval 0-20 ps. The fitting of the simulation data to the three different models $\mathrm{A}, \mathrm{B}$ and $\mathrm{C}$ and the corresponding regression coefficient are also shown. 


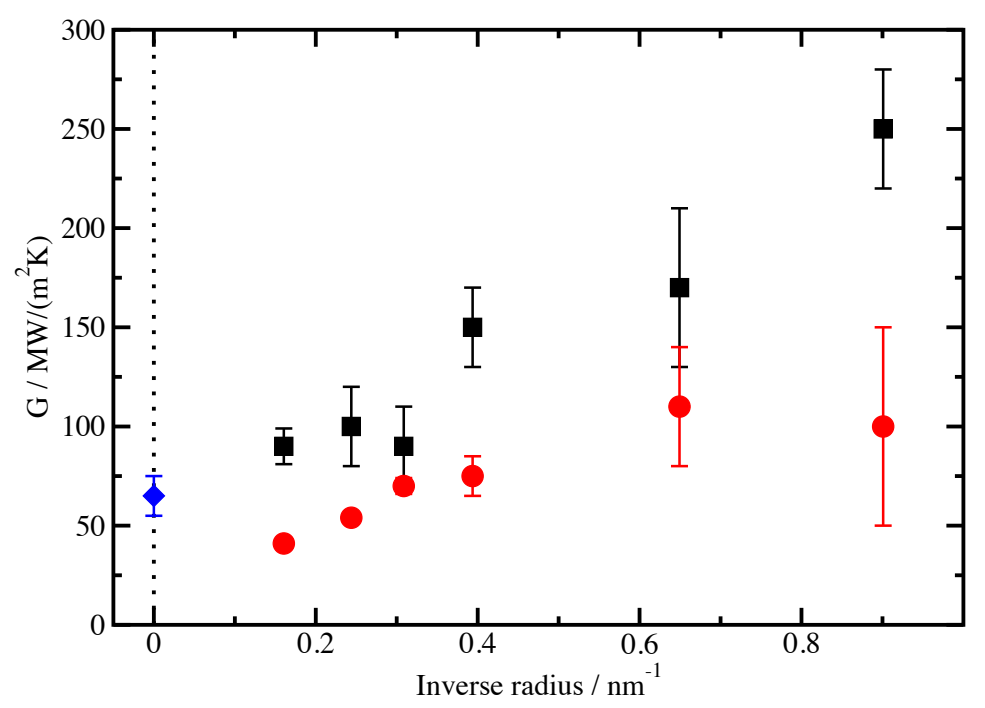

Figure 4: Dependence of the thermal conductance with the nanodroplet diameter for models A (circles) and $\mathrm{C}$ (squares). The diamond represents an estimate of the thermal conductance of the octane-water planar interface using an stationary non equilibrium molecular dynamics approach $[10]$.

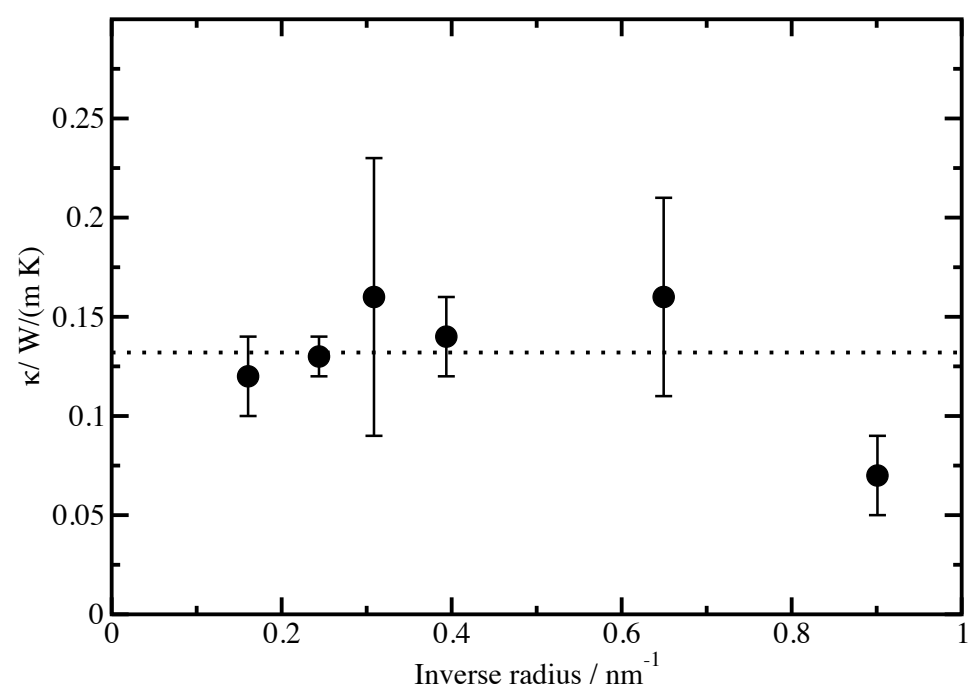

Figure 5: Dependence of the thermal conductivity with the nanodroplet size for model C (symbols). The dotted line represents the experimental thermal conductivity of $n$-decane at $300 \mathrm{~K}$ [44]. 